\title{
DIFFERENCES IN THE COMPOSITION OF CULTIVABLE AEROBIC AND FACULTATIVE ANAEROBIC ORAL MICROBIOTA IN CATS OF VARIOUS AGE GROUPS
}

\author{
Sondorová, M., Koščová, J., Kačírová, J., Mad’ar, M. \\ Department of Microbiology and Immunology, \\ University of Veterinary Medicine and Pharmacy in Košice, Komenského 73, 04181 Košice \\ Slovakia
}

miriam.sondorova@gmail.com

\section{ABSTRACT}

The feline oral cavity is naturally inhabited by various microorganisms contributing to the maintenance of its oral health. The imbalance of oral microbiota or the presence of pathogenic agents can lead to secondary oral diseases. Various factors such as sex, diet, breed, environment and even age, affect the composition of a healthy oral microbiota during the life of cats. The purpose of this study was to compare the composition of culturable aerobic and facultative anaerobic microbiota in cats in terms of different age categories. We used conventional cultivation methods in conjunction with microscopic and biochemical methods to isolate and identify the microorganisms found in the oral cavity of cats. The examination of 76 samples confirmed the dominance of the phylum Proteobacteria in almost all groups. Commonly occurring bacteria have been identified, i.e. Streptococcus spp., Neisseria spp. and Pasteurella spp. Although aerobic and facultative anaerobic oral microbiota were examined, differences between age groups were noted. The microbial diversity of the oral microbiota significantly increased with age.

Key words: age groups; cultivation methods; feline; oral microbiota

\section{INTRODUCTION}

The oral cavity is the first part of the gastrointestinal tract where the process of digestion begins, and therefore it creates a space for the action of various microorganisms [8]. The constant flow of saliva and the unique biological properties of each part of the oral cavity provide a place for attachment of microorganisms [22]. In particular, the surface of the teeth has the highest microbial load compared to other surfaces [25]. Complex, multi-species communities of resident microorganisms, from aerobic to anaerobic, play a crucial role in oral health and diseases. Important is 
the beneficial defensive function or rather the competition with the pathogenic bacteria introduced with food or from the external environment [15].

The feline oral microbiota consists of several bacterial phyla such as: Proteobacteria, Bacteroidetes, Firmicutes, Actinobacteria, Fusobacteria and others [4]. Differences in the diversity and dominance of bacterial strains have been reported in terms of health and diseases [22], and it has also been disclosed that the diversity of microbiota in the oral cavity of cats is significantly greater than that in dogs $[11,15]$.

The feline oral microbiota may pose a risk of systemic disease if it is transmitted to other sites in the body, e.g. in the case of aspiration pneumonia [1]. Microorganisms can also be transmitted by biting, thus causing infections of wounds usually described in humans [9]. The feline oral microbiota can be a source of zoonotic pathogens (e.g. Pasteurella spp.), not only in terms of bites [17], but also by the potential transmission of periodontal bacteria to the oral cavity of cat owners [2].

Microbial communities inhabiting the oral cavity can be affected by various factors such as sex, environment, breed [13], diet [1] and age [13, 19]. Also, the composition of the oral microbiota may vary with the disease. Differences in compositions of the oral microbiota between healthy cats and those with a disease have been reported for periodontal disease (PD) $[13,16]$, feline chronic gingivostomatitis (FCGS) $[6,12]$ and feline immunodeficiency virus (FIV) [10, 14, 23], where the predominance of certain oral bacteria may cause or contribute to the development of the disease [18].

For the detection of feline oral microbiota, culture-independent methods (gene sequence analyses) have been increasingly used as less time-consuming than the culture methods [3]. However, conventional culture methods are still essential for the isolation and identification of microorganisms [8].

Natural colonization of the oral cavity by microorganisms occurs from birth [19]. With increasing age, the diversity of microbial communities increases significantly [ 1 , 22] which correlates with the changes during important life stages [21]. Only a few studies have been involved in investigating the effects of age on the oral microbiota in cats. Therefore, the aim of our study was to evaluate on the basis of biochemical methods and cultures how the age of cats can affect the composition of their oral microbiota.

\section{MATERIALS AND METHODS}

\section{Age groups of cats}

An important step before sampling was the formation of age groups. Based on the scheme proposed by $\mathrm{V}$ o g t et al. [21], the following age groups were formed: kittens (from birth until 5 months), young adults (6 months to 2 years), adults ( 3 years to 7 years) and seniors (over 8 years).

\section{Sample collection}

Samples were obtained from 76 clinically healthy European Shorthair cats by inserting a sterile swab into the cat's mouth and rotating the swab for approximately $10-15$ seconds. The swab included the entire oral cavity: the upper and lower dental arcade (supragingival plaque), gums, labial and buccal mucosa, palatal mucosa and sublingual region. The swabs were then placed in Amies transport medium (Sarstedt, Nümbrecht, Germany) at $8{ }^{\circ} \mathrm{C}$ and

Table 1. Characterization of feline age groups

\begin{tabular}{lcccc}
\hline \multicolumn{1}{c}{ Age groups } & Mean age & $\begin{array}{c}\text { Sex F/M } \\
{[\%]}\end{array}$ & $\begin{array}{c}\text { Environment I/O/C } \\
{[\%]}\end{array}$ & $\begin{array}{c}\text { Diet D/W/C } \\
{[\%]}\end{array}$ \\
\hline Kittens $(\mathrm{n}=20)$ & 2 months & $60 / 40$ & $75 / 20 / 5$ & $0 / 25 / 75$ \\
Young adults $(\mathrm{n}=20)$ & 1.5 years & $50 / 50$ & $50 / 25 / 25$ & $0 / 0 / 100$ \\
Adults $(\mathrm{n}=20)$ & 4 years & $55 / 45$ & $35 / 35 / 30$ & $0 / 0 / 100$ \\
Seniors $(\mathrm{n}=16)$ & 11 years & $62.5 / 37.5$ & $12.5 / 25 / 62.5$ & $0 / 6.2 / 93.8$ \\
\hline
\end{tabular}

F-female; M-male; I-indoor; O-outdoor; C-combination; D-dry; W-wet 
processed within 24 hours. The health status of each cat was assessed on the basis of anamnesis, clinical examination and oral health (normal gingiva with sharp, non-inflamed edges).

All procedures involving animals followed the guidelines stated in the Guide for the Care and Use of Animals (Protocol No. 3323/16-221/3), approved by the State Veterinary and Food Administration of the Slovak Republic and by the Ethics Commission of the University of Veterinary Medicine and Pharmacy in Košice, Slovakia. The animals were handled in a humane manner in accordance with the guidelines established by the relevant commissions. All applicable international, national and institutional guidelines for the care and use of animals were observed. The basic data about the cats were obtained from cat owners as well as their consent to participate in the study. A summary of the data obtained on the basis of age grouping may be seen in Table 1.

\section{Bacterial analysis of samples}

Each swab was plated on blood agar, MacConkey agar (HiMedia, Mumbai, India) and Endo agar (HiMedia, Mumbai, India). The blood agar was prepared as Tryptone Soya Agar (HiMedia, Mumbai, India) supplemented with $5 \%$ of sterile defibrinated sheep blood. The inoculated agar plates were then incubated under aerobic conditions at $37^{\circ} \mathrm{C}$ for 24 hours. After incubation, the plates were examined and then different types of colonies were subcultured to obtain pure cultures. The presence of haemolytic activity and morphological characteristics such as growth, shape, size and colour of pure cultures were evaluated on blood agar. In addition to culture examinations, Gram staining was used to differentiate Gram-positive and Gram-negative bacteria and also to determine the microscopic morphology.

Bacterial isolates were then subjected to biochemical assays for oxidase and catalase activity. The indole and urease tests were used to differentiate Pasteurella species. Final biochemical identification of strains were done using commercial identification kits: STAPHYtest 24; STREPTOtest 24; NEFERMtest 24; ENTEROtest 16; ENTEROtest 24 (Erba Lachema, Brno, Czech Republic). Subsequently, a specific bacterial strain was determined using the software Program TNW ProAuto 7.0. The overall results were evaluated and analysed by statistical-mathematical methods in percentage terms using the Microsoft Excel programme.
Two selected isolates of Neisseria spp. were identified by genotyping for their species identification. DNA was isolated using DNAzol direct (Molecular Research Center Inc., Cincinnati, USA) according to the manufacturer's instructions. The $16 \mathrm{~S}$ ribosomal RNA (rRNA) genes from the isolates were amplified by PCR using the universal primers 27F (5'-AGAGTTTGATCMTGGCTCAG-3') and 1492R (5'-CGGYTACCTTGTTACGACTT-3'). The PCR cycling conditions comprised an initial denaturation phase of $5 \mathrm{~min}$ at $94^{\circ} \mathrm{C}$, followed by 30 cycles of denaturation at $94^{\circ} \mathrm{C}$ for $1 \mathrm{~min}$, annealing at $55^{\circ} \mathrm{C}$ for $1 \mathrm{~min}$ and primer extension at $72{ }^{\circ} \mathrm{C}$ for $3 \mathrm{~min}$ and finally a primer extension step at $72{ }^{\circ} \mathrm{C}$ for $10 \mathrm{~min}$. The PCR was performed in a thermal cycler (TProfesional Basic, Biometra GmbH, Göttingen, Germany), aliquot PCR products were separated by horizontal $3 \%(\mathrm{w} / \mathrm{v})$ agarose gel electrophoresis in Tris-acetate-EDTA buffer ( $\mathrm{pH}$ 7.8) and visualized with GelRed (Biotium, Inc., Hayward, CA, USA) under ultraviolet light. The amplification products were sent for purification and sequencing using primer 1492R (Microsynth, Wien, Austria).

\section{RESULTS}

The combination of culture evaluations and subsequent microscopic findings were the first steps in identifying most of the representatives of the oral microbiota at the level of the bacterial genus. A total of 18 different bacterial genera were isolated from the 76 samples. The most frequently isolated bacterial genera were Streptococcus (23.9\%), Pasteurella (22.4\%) and Neisseria (17.9\%). Pasteurella spp. were identified on the basis of Gram staining (Gram-negative coccobacilli), absence of haemolysis on blood agar, grey and viscous colonies, absence of growth on MacConkey and Endo agar, and positive biochemical activity of catalase and oxidase tests. Only two isolates of Pasteurella spp. have been accurately identified as Pasteurella multocida and Pasteurella pneumotropica based on their different biochemical characteristics (Table 2).

Escherichia coli was identified by its typical green metallic sheen on Endo agar, and some suspect isolates were confirmed by biochemical assays. Neisseria was identified based on the culture's characteristics (round shape, opaque, shiny, smooth colonies), microscopic morphology (Gram-negative coccoid or short rods which often oc- 
Table 2. Biochemical characterization of two Pasteurella isolates

\begin{tabular}{lcccc}
\hline & Oxidase & Catalase & Urease & Indole \\
\hline $\begin{array}{l}\text { Pasteurella multocida } \\
\text { Pasteurella } \\
\text { pneumotropica }\end{array}$ & + & + & - & + \\
\hline
\end{tabular}

+- positive; - - negative curred in pairs), positive catalase activity and positive oxidase activity. However, two isolates of Neisseria spp. were also subjected to sequencing analysis (16S rRNA) and were identified as Neisseria zoodegmatis and Neisseria animaloris. Both species formed non-haemolytic, round shaped, yellowish to white colonies on blood agar (Fig. 1).

The results of biochemical commercial tests of all identified species are shown in Table 3. A score of less than $50 \%$ is reported only at the level of the bacterial genus.

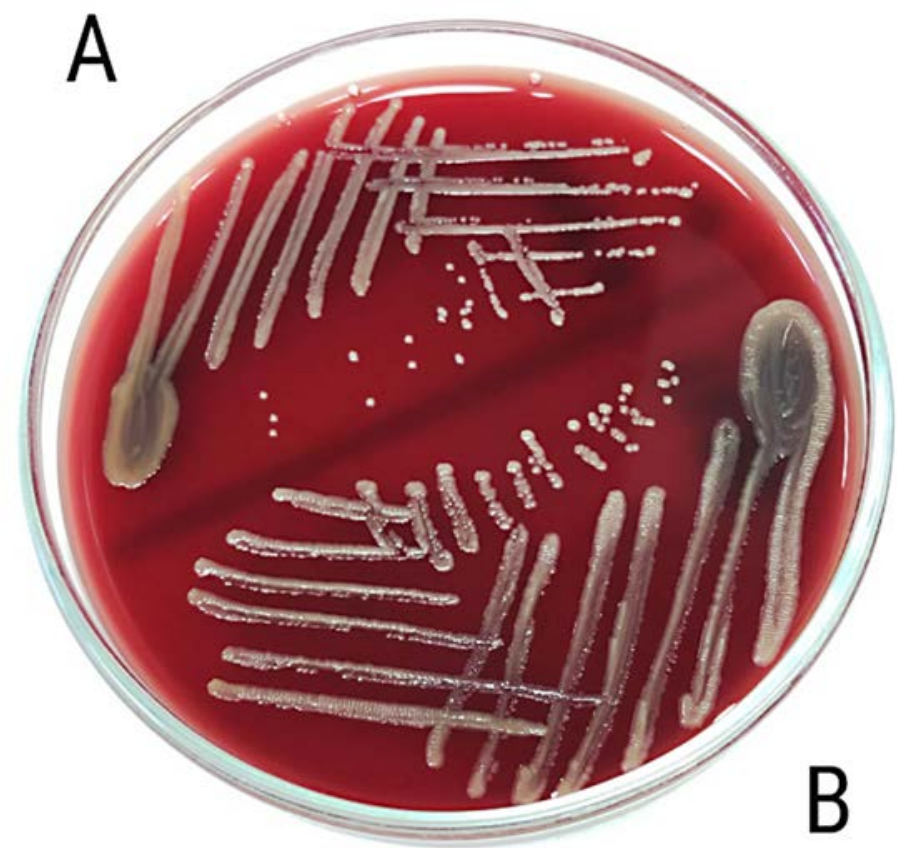

Fig. 1. Growth of Neisseria zoodegmatis and Neisseria animaloris on blood agar A-Neisseria zoodegmatis; $\mathbf{B}-$ Neisseria animaloris

The main aim of this study was to characterize and compare the representatives of cultivable aerobic and facultative anaerobic oral microbiota of cats between age groups (Fig. 2). The members of the genus Streptococcus $(40.7 \%)$ in the group of kittens were predominantly identified, followed by Pasteurella spp. (33.3\%) and Staphylococcus spp. (11.1\%). In the case of young adults, the diversity of the bacteria was higher. The genera found in the highest proportions were Pasteurella (25.7\%) and Streptococcus (25.7\%), followed by Moraxella (8.6\%) and Neisseria $(8.6 \%)$. In the adult cats, the most frequently isolated bacteria were Streptococcus spp. (29.7 \%) and Neisseria spp.
(27\%). The genus Pasteurella (16.8\%) was the third most frequently identified. The highest bacterial diversity was recorded in the group of seniors. The predominant genus was Neisseria spp. (28.6\%) followed by Pasteurella spp. (17.1\%) and Staphylococcus spp. (11.4\%). The percentage comparison of bacterial phyla between age groups can be seen in Fig. 3.

Other bacterial genera detected in young adults were: Enterobacter, Kocuria; other bacterial genera detected in seniors were: Aeromonas, Brevundimonas, Corynebacterium, and Sutonella, Weeksella. 
Table 3. Bacterial species identified by commercial identification kits

\begin{tabular}{|c|c|c|}
\hline Bacterial species & Number of isolates & $\begin{array}{l}\text { Average Percentage } \\
\text { Identification Score [\%] }\end{array}$ \\
\hline Aerococcus spp. & 1 & 48.15 \\
\hline Aerococcus viridans & 2 & 95.11 \\
\hline Aeromonas caviae & 1 & 90.33 \\
\hline Aeromonas ichthiosmia & 1 & 100 \\
\hline Brevundimonas vesicularis & 1 & 78.12 \\
\hline Citrobacter braaki & 2 & 95.38 \\
\hline Enterobacterspp. & 3 & 48.97 \\
\hline Escherichia coli & 3 & 99.76 \\
\hline Klebsiella oxytoca & 2 & 99.34 \\
\hline Kocuria kristinae & 1 & 99.02 \\
\hline Moraxella osloensis & 2 & 54.69 \\
\hline Moraxella spp. & 7 & 49.88 \\
\hline Shewanella algae & 2 & 92.11 \\
\hline Staphylococcus felis & 1 & 95.11 \\
\hline Staphylococcus hominis ssp. hominis & 2 & 99.80 \\
\hline Staphylococcus sciuri & 2 & 100 \\
\hline Staphylococcus simulans & 1 & 60.24 \\
\hline Staphylococcus spp. & 4 & 49.74 \\
\hline Staphylococcus warneri & 1 & 99.16 \\
\hline Streptococcus sanguinis/parasanguinis & 17 & 94.77 \\
\hline Streptococcus spp. & 8 & 49.52 \\
\hline Streptococcus suis & 2 & 99.78 \\
\hline Streptococcus uberis & 5 & 99.90 \\
\hline Sutonella indologenes & 2 & 99.38 \\
\hline Weeksella virosa & 1 & 92.35 \\
\hline
\end{tabular}

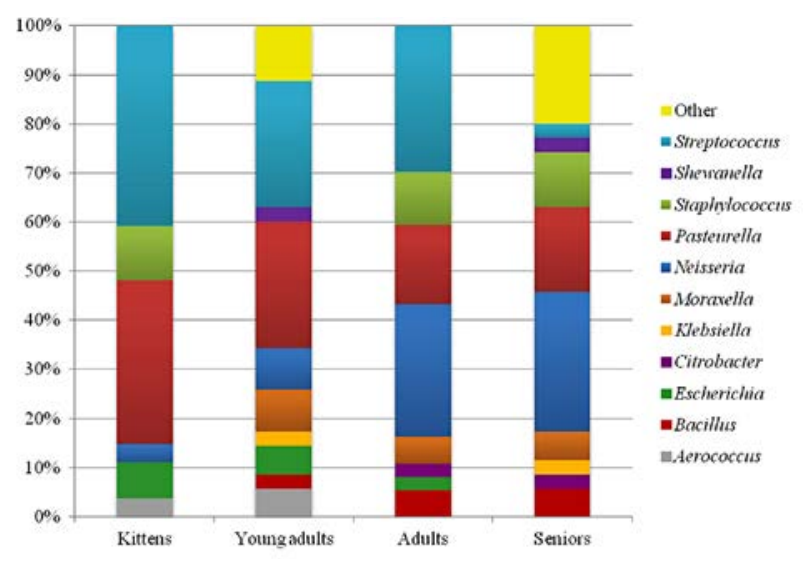

Fig. 2. Comparison of the occurrence of bacterial genera between age groups of cat

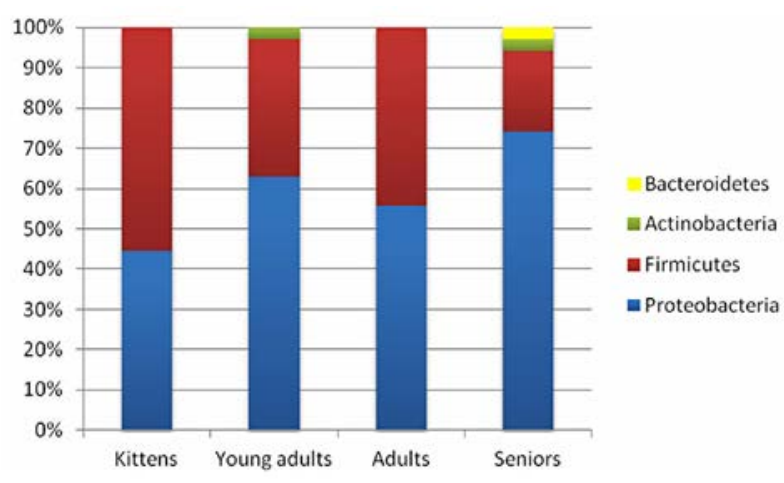

Fig. 3. Comparison of bacterial phyla between age groups 


\section{DISCUSSION}

The composition of a culturable feline oral microbiota may be influenced by many factors. This study focused on various stages in a cat's life and the potential differences in the diversity of oral microbiota in the different age groups.

Oral swabs were performed without anaesthesia or sedation because the cats were calm during swabbing. This approach differed from that used in other studies $[4,18$, 24] when samples were taken during anaesthesia as a part of routine dental examination. Subsequent bacteriological examinations focused mainly on the isolation and identification of the facultative anaerobic and aerobic bacteria, as the samples were transported over longer distances to the laboratory. Also, in the study by $\mathrm{R} \mathrm{a} \mathrm{z} \mathrm{a} \mathrm{li} \mathrm{et} \mathrm{al.} \mathrm{[17]}$ anaerobic bacteria were not included in the investigation of the oral microbiota in the cats, as anaerobic bacteria require more complex cultivation and immediate processing after sampling.

The current trend in the analysis of the oral microbiota of cats is carried out mainly by NGS sequencing, so that the samples are subjected directly to DNA extraction without previous conventional culture $[5,20]$. In addition, compared to standard culture methods, a culture-independent methodology can detect also the presence of non-culturable and unknown microorganisms in the oral microbiota [3]. On the other hand, from the point of view of veterinary practice, culture methods are used in conjunction with the determination of the antibiotic susceptibility of oral bacteria which may cause either primary or secondary infections.

Commonly occurring bacteria, for example Streptococcus, Neisseria and Pasteurella, were identified in this study by culture and biochemical identifications. The dominance of the Proteobacteria phylum was also noted. A study of the oral microflora of cats with or without disease showed that a-haemolytic Streptococci were the most common genus in healthy cats while in the sick cats, the genus Staphylococcus dominated [24]. S t u r g e o n et al. [20] investigated the oral microbiota from 11 clinically healthy cat and detected the predominance of Proteobacteria phylum, mainly the genera Moraxella, Pasteurella, Neisseria and unclassified bacteria of the Pasteurellaceae family. Another study reported that the most commonly identified genera in healthy cats were, Enhydrobacter, Moraxella and Capnocytophaga [18]. The bacterial genera Moraxella and Neisse- ria were obtained from a healthy feline oral cavity. These were also described as two of the most common aerobic genera identified in samples from humans bitten by cats [3]. $\mathrm{H}$ a r ris et al. [7] reported that the most dominant phylum in their study of the oral cavity of healthy cats was Bacteroidetes, which does not agree with our results. This may be related to other factors that may affect the composition of the oral microbiota [13].

The research of Spears et al. [19] focused on the development of oral microbiota in kittens from birth to 8 weeks of age. They examined a total of 5 litters; the number of individuals was 16. Phylogenetic analysis during their growth showed an increase in the proportion of phyla. Although a different age range was used in our study, our results indicated an increase in bacterial diversity.

\section{CONCLUSIONS}

Our study investigated changes in the composition of the oral microbiota during the life of cats. Despite the limitations of culture and biochemical methods, our results agree with the previous studies regarding the abundance and predominance of the Proteobacteria strains in the oral microbiota of cats. An increase in the bacterial diversity with age was observed in the older age groups. The difference in the oral microbiota may have been due to changes during the life stages, which can be associated with the change of the diet from milk to commercial or other available food for kittens. Further studies are needed to demonstrate the potential effects of other factors. A study of cultivable healthy oral microbiota of cats with respect to age can differentiate potential pathogenic bacteria not only for the cats themselves but also for the potential risk of bite infections in humans.

\section{ACKNOWLEDGEMENTS}

This study was supported by the Slovak Research and Development Agency under the contract No. APVV-16-0171 and by the Scientific Grand Agency of Ministry of Education of the Slovak Republic under the contract No. VEGA 1/0788/19. 


\section{REFERENCES}

1. Adler, C. J., Malik, R., Browne, G. V., Norris, J. M., 2016: Diet may influence the oral microbiome composition in cats. Microbiome, 4, 23, 1-9. DOI: 10.1186/s40168-016-0169-y.

2. Booij-Vrieling, H. E., van der Reijden, W. A., Houwers, D. J., de Wit, W. E. A. J., Bosch-Tijhof, C. J., Penning, L. C., et al., 2011: Comparison of periodontal pathogens between cats and their owners. Vet. Microbiol., 144, 147-152. DOI: 10.1016/j.vetmic.2009.12.046.

3. Davis, E. M., 2016: Gene sequence analyses of the healthy oral microbiome in humans and companion animals: A comparative review. J. Vet. Dent., 33, 2, 97-107. DOI: 10.1177/0898 756416657239 .

4. Dewhirst, F. E., Klein, E. A., Bennett, M., Croft, J. M., Harris, S. J., Marshall-Jones, Z. V., 2015: The feline oral microbiome: a provisional 16S rRNA gene based taxonomy with full-length reference sequences. Vet. Microbiol., 175, 294303. DOI: 10.1016/j.vetmic.2014.11.019.

5. Dolieslager, S. M., Riggio, M. P., Lennon, A., Lappin, D. F., Johnston, N., Taylor, D., et al., 2011: Identification of bacteria associated with feline chronic gingivostomatitis using culture-dependent and culture-independent methods. Vet. Microbiol., 148, 93-98. DOI: 10.1016/j.vetmic.2010.08.002.

6. Dolieslager, S. M., Bennet, D., Johnston, N., Riggio, M. P., 2013: Novel bacterial phylotypes associated with the healthy feline oral cavity and feline chronic gingivostomatitis. Res. Vet. Sci., 93, 3, 428-432. DOI: 10.1016/j.rvsc.2012.11.003.

7. Harris, S., Croft, J., O’Flynn, C., Deusch, O., Colyer, A., Allsopp, J., et al., 2015: A pyrosequencing investigation of differences in the feline subgingival microbiota in health, gingivitis and mild periodontitis. PLOS ONE, 10, 11, e0136986. DOI: 10.1371/journal.pone.0136986.

8. Kil, D. Y., Swanson, K. S., 2011: Companion animals symposium: Role of microbes in canine and feline health. J. Anim. Sci., 89, 5, 1498-1505. DOI: 10.2527/jas.2010-3498.

9. Magaji, A. A., Saulawa, M. A., Salihu, M. D., Junaidu, A. U., Shittu, A., Gulumbe, M. L., et al., 2008: Oral microflora of stray domestic cats (Felis catus) found in the premises of two human hospitals in Sokoto, Nigeria. Sokoto J. Vet. Sci., $7,1,9-12$.

10. Miller, C., Boegler, K., Carver, S., MacMillan, M., Bielefeldt-Ohmann, H., VandeWoude, S., 2017: Pathogenesis of oral FIV infection. PLOS ONE, 12, 9, e0185138. DOI: 10.1371/ journal.pone.0185138.

11. Misic, A. M., Davis, M. F., Tyldsley, A. S., Hodkinson, B. P.,
Tolomeo, P., Hu, B., et al., 2015: The shared microbiota of humans and companion animals as evaluated from Staphylococcus carriage sites. Microbiome, 3, 2, 1-19. DOI: 10.1186/ s40168-014-0052-7.

12. Nakanishi, H., Furuya, M., Soma, T., Hayashiuchi, Y., Yoshiuchi, R., Matsubayashi, M., et al., 2019: Prevalence of microorganisms associated with feline gingivostomatitis. J. Feline Med. Surg., 21, 2, 103-108. DOI: 10.1177/1098612X 18761274.

13. Older, C. E., Diesel, A. B., Lawhon, S. D., Queiroz, C. R. R., Henker, L. C., Hoffmann, A. R., 2019: The feline cutaneous and oral microbiota are influenced by breed and environment. PLOS ONE, 14, 7, e0220463. DOI: 10.1371/journal. pone. 0220463 .

14. Older, C. E., Gomes, M. O. S., Hoffmann, A. R., Policano, M. D., Reis, C. A. C. D., Carregaro, A. B., et al., 2020: Influence of the FIV status and chronic gingivitis on feline oral microbiota. Pathogens, 9, 5, 1-11. DOI: 10.3390/pathogens 9050383.

15. Özavci, V., Erbas, G., Parin, U., Yüksel, H. T., Kirkan, Ş., 2019: Molecular detection of feline and canine periodontal pathogens. Vet. Anim. Sci., 8, 100069. DOI: 10.1016/j.vas. 2019.100069.

16. Pérez-Salcedo, L., Herrera, D., Esteban-Saltiveri, D., León, R., Jeusette, I., Torre, C., et al., 2011: Comparison of two sampling methods for microbiological evaluation of periodontal disease in cats. Vet. Microbiol., 149, 500-503. DOI: 10. 1016/j.vetmic.2010.11.021.

17. Razali, K., Kaidi, R., Abdelli, A., Menoueri, M. N., Ait-Oudhia, K., 2020: Oral flora of stray dogs and cats in Algeria: Pasteurella and other zoonotic bacteria. Vet. World, 13, 12, 2806-2814. DOI:10.14202/vetworld.2020.2806-2814.

18. Rodrigues, M. X., Bicalho, R. C., Fiani, N., Lima, S. F., Peralta, S., 2019: The subgingival microbial community of feline periodontitis and gingivostomatitis: characterization and comparison between diseased and healthy cats. Sci. Rep., 9, 1, 12340. DOI: 10.1038/s41598-019-48852-4.

19. Spears, J. K., Vester Boler, B., Gardner, C., Li, Q., 2017: Development of the oral microbiome in kittens. In Companion Animal Nutrition (CAN) Summit: The Nexus of Pet and Human Nutrition: Focus on Cognition and Microbiome. Helsinki, Finland, May 4-7, 73-81.

20. Sturgeon, A., Pinder, S. L., Costa, M. C., Weese, J. S., 2014: Characterization of the oral microbiota of healthy cats using next-generation sequencing. Vet. J., 201, 2, 223-229. DOI: 10.1016/j.tvjl.2014.01.024. 
21. Vogt, A. H., Rodan, I., Brown, M., Brown, S., Buffington, C. A., Forman, M. J. L., et al., 2010: AAFP-AAHA: feline life stage guidelines. J. Feline Med. Surg., 12, 43-54. DOI: 10. 1016/j.jfms.2009.12.006.

22. Wallis, C. V., Marshall-Jones, Z. V., Deusch, O., Hughes, K. R., 2017: Canine and Feline Microbiomes. In Singh, R., Kothari, R., Koringa, P., Singh, S.: Understanding Host-Microbiome Interactions-An Omics Approach. Springer, Singapore, 279-325. DOI: 10.1007/978-981-10-5050-3_17.
23. Weese, S. J., Nichols, J., Jalali, M., Litster, A., 2015: The oral and conjunctival microbiotas in cats with and without feline immunodeficiency virus infection. Vet. Res., 46, 21-31. DOI: 10.1186/s13567-014-0140-5.

24. Whyte, A., Gracia, A., Bonastre, C., Tejedor, M. T., Whyte, J., Monteagudo, L. V., et al., 2017: Oral disease and microbiota in free-roaming cats. Top. Companion Anim. Med., 32, 3, 91-95. DOI: 10.1053/j.tcam.2017.07.003.

25. Zambori, C., Tirziu, E., Nichita, I., Cumpanasoiu, C., Gros, R. V., Seres, M., et al., 2012: Biofilm implication in oral diseases of dogs and cats. Anim. Sci. Biotechnol., 45, 2, 208-212.

Received January 14, 2021

Accepted February 16, 2021 